\title{
Quantum entanglement and entropy
}

\author{
Filippo Giraldi ${ }^{1,2,3}$ and Paolo Grigolini ${ }^{1,2,3}$ \\ ${ }^{1}$ Dipartimento di Fisica dell'Università di Pisa and INFM, Piazza Torricelli 2, 56127 Pisa, Italy \\ ${ }^{2}$ Istituto di Biofisica CNR, Area della Ricerca di Pisa, Via Alfieri 1, San Cataldo 56010 Ghezzano-Pisa, Italy \\ ${ }^{3}$ Center for Nonlinear Science, University of North Texas, P.O. Box 311427, Denton, Texas 203-1427
}

(Received 30 January 2001; published 20 August 2001)

\begin{abstract}
Entanglement is the fundamental quantum property behind the now popular field of quantum transport of information. This quantum property is incompatible with the separation of a single system into two uncorrelated subsystems. Consequently, it does not require the use of an additive form of entropy. We discuss the problem of the choice of the most convenient entropy indicator, focusing our attention on a system of two qubits, and on a special set, denoted by $\mathfrak{I}$. This set contains both the maximally and partially entangled states that are described by density matrices diagonal in the Bell basis set. We select this set for the main purpose of making our work of analysis more straightforward. As a matter of fact, we find that in general the conventional von Neumann entropy is not a monotonic function of the entanglement strength. This means that the von Neumann entropy is not a reliable indicator of the departure from the condition of maximum entanglement. We study the behavior of a form of nonadditive entropy, made popular by the 1988 work by Tsallis [J. Stat. Phys. 52, 479 (1988)]. We show that in the set $\mathfrak{I}$, implying the key condition of nonvanishing entanglement, this nonadditive entropy indicator turns out to be a strictly monotonic function of the strength of the entanglement, if entropy indexes $q$ larger than a critical value $Q$ are adopted. We argue that this might be a consequence of the nonadditive nature of the Tsallis entropy, implying that the world is quantum and that uncorrelated subsystems do not exist.
\end{abstract}

DOI: 10.1103/PhysRevA.64.032310 PACS number(s): 03.67.-a, 03.65.Ta, 05.20. $-\mathrm{y}, 05.30 .-\mathrm{d}$

\section{INTRODUCTION}

Entanglement is the fundamental quantum property behind the interesting process of quantum teleportation proposed some years ago by Bennett et al. [1]. For this reason it is important to quantify entanglement [2]. It was also found that the fidelity of the quantum teleportation is always larger than that of any classical communication protocol, even in the noisy environment [3]. However, for the teleportation to take place perfectly, it is necessary for the sender and the recipient [1] to share a maximally entangled state. This generates the need for special purification protocols. On the other hand, a statistical analysis of these protocols shares deep similarities with the second principle of thermodynamics [4-6].

In spite of the plausible conjecture that there exists a deep connection between quantum teleportation and thermodynamics [4-6], the entanglement is expressed by means of an entropic structure, the conventional von Neumann entropy, only in the case of pure states. In general the definitions of entanglement dictated by the purification protocol are not directly related to entropy indicators. Cerf and Adami [7] showed that the conditional von Neumann entropy can become negative, thereby pointing out the nonordinary information aspects of quantum entanglement. However, the reason for the lack of a direct connection between quantum entanglement of mixed states and the entropy indicator is probably that, as shown in this paper, the von Neumann entropy is not a reliable indicator of entanglement [7]. The inadequacy of the Shannon information, and consequently of the von Neumann entropy as an appropriate quantum generalization of Shannon entropy [8], was already pointed out by Brukner and Zeilinger $[9,10]$. It is notable that according to these authors a new kind of entropy indicator is made necessary by the following deep difference between quantum and classical information. In classical measurement the Shannon information is a natural measure of our ignorance about the properties of a system, whose existence is independent of measurement. Quantum measurement, conversely, cannot be claimed to reveal the properties of a system that existed before the measurement was made.

In this paper we focus on the earlier mentioned quantum property, essential for teleportation of information: entanglement. Entanglement implies that a system cannot be divided into two uncorrelated subsystems; this, in turn, makes useless the ordinary request for an additive form of entropy. Thus in this paper we explore and discuss the possible benefits stemming from the adoption of the nonadditive entropy indicator advocated years ago by Tsallis [11]. The choice of the Tsallis form of nonadditive entropy for systems that cannot be divided in uncorrelated subsystems (the nonextensive case) was made compelling by recent work of Abe [12]. This is so because this author proved that the three axioms making the Shannon entropy a unique form of the extensive condition can be properly generalized so as to make the Tsallis entropy a unique form of the nonextensive case.

The Tsallis entropy is applied to a large number of physical conditions, characterized by the existence of extended correlation [13]. The use of this form of nonadditive indicator in the field of quantum teleportation, to the best of our knowledge, was discussed in only a few papers [14-17]. Reference [14] claimed to the realization of a greater sensitivity to the occurrence of a dephasing process, resulting in the annihilation of any form of entanglement. References [15-17] pointed out the efficiency of Tsallis entropy for the detection of the breakdown of local realism. Reference [16] aimed at proving that the Jaynes principle $[18,19]$, applied to 
the nonextensive entropy, naturally yields an entangled state. The present paper, although based on an adoption of the Tsallis nonextensive entropy as in Ref. [16], adopts a quite different perspective, which does not rest on the adoption of the Jaynes principle.

The outline of the paper is as follows. Section II is devoted to a concise illustration of the main properties of the nonextensive entropy at work in this paper. Section III, devoted to the entanglement of formation, shows that the von Neumann entropy, in general, is not a monotonic function of entanglement, while the nonextensive entropy is a monotonically increasing function of the entanglement for suitably large values of the entropy index. In Sec. IV we study the entanglement of a de-phasing process, and we extend the monotonic properties of nonextensive entropy to a condition more general than that of Sec. III. Section IV is devoted to concluding remarks.

\section{NONEXTENSIVE ENTROPY}

The entropy indicator applied in this paper has the form

$$
S_{q}(\rho) \equiv \operatorname{Tr} \frac{\rho-\rho^{q}}{q-1}
$$

This form was originally proposed by Tsallis [11] for the purpose of establishing the most convenient thermodynamic perspective for fractal processes. It is worth remarking that this is a generalization of the conventional Gibbs-Shannon entropy indicator, whose explicit form is recovered from Eq. (1) in the limiting case $q \rightarrow 1$. This entropy indicator does not fit the additivity condition, namely, the requirement that the entropy of a system $A+B$, consisting of two statistically independent subsystems $A$ and $B$, be the sum of the entropies of these two subsystems. In fact, the definition of Eq. (1) yields, in this case, the equality

$$
S_{q}(A+B)=S_{q}(A)+S_{q}(B)+(1-q) S_{q}(A) S_{q}(B),
$$

making it evident that the additive property is recovered only in the case $q=1$, which, as noted earlier, makes the nonextensive entropy of Eq. (1) become equivalent to the usual Shannon entropy.

According to Tsallis and to advocates of this nonextensive entropy indicator (for a review, see Ref. [13]), the violation of the additive condition turns into a benefit when this entropy indicator is used to study cases where the ideal condition of statistical independence is prevented by the nature of the processes under study [13]. Notable examples are processes with long-range correlation [13]. We believe that quantum entanglement, which is the basic property for teleportation [1], is probably the most evident example of a condition incompatible with the existence of uncorrelated subsystems. For this reason, the additivity condition can be safely renounced, and the adoption of an entropy index $q$ $\neq 1$ might turn out to be beneficial. This paper is devoted to discussing to what extent this conjecture proves to be correct.

\section{TSALLIS ENTROPY AT WORK: ENTANGLEMENT OF FORMATION}

In this section we derive the central result of this paper. We show that in the case of initial and final states, both with nonvanishing entanglement, and described by a statistical density matrix diagonal in the Bell basis, the Tsallis entropy decreases upon an increase of the entanglement of formation. The results of this section refer to the entanglement of formation [20] of a system of two qubits. Thus, to make this paper as self contained as possible, here we give a concise illustration of this key measure of entanglement. The main point is that the entanglement property is defined without any ambiguity for pure states [1]. The entanglement of formation extends to the statistical case the ordinary definition as follows. Let us denote by $\rho$ the statistical density matrix for the mixed state of a space $S_{1 / 2}^{(1)} \times S_{1 / 2}^{(2)}$ of two spin-1/2 particles. The entanglement of formation [21], denoted by the symbol $E_{F}$ throughout, is defined as the minimum average entanglement of every ensemble of pure states that represents $\rho$,

$$
E_{F}(\rho)=\min _{\rho=\Sigma_{i} P_{i}\left|\alpha_{i}\right\rangle\left\langle\alpha_{i}\right|} \sum_{i} P_{i} E\left(\left|\alpha_{i}\right\rangle\right),
$$

where $E\left(\left|\alpha_{i}\right\rangle\right)$ denotes the entanglement of a pure state, which is defined according to the usual prescription [1] by the expression

$$
E(|\alpha\rangle)=-\operatorname{Tr}\left(\rho_{A} \log _{2} \rho_{A}\right)=-\operatorname{Tr}\left(\rho_{B} \log _{2} \rho_{B}\right),
$$

with

$$
\rho_{A} \equiv \operatorname{Tr}_{2} \rho, \quad \rho_{B} \equiv \operatorname{Tr}_{1} \rho .
$$

Wootters, in an enlightening paper [20], derived an explicit formula for the entanglement of formation of any arbitrary mixed state of a system of two qubits. This formula reads

$$
E_{F}(\rho)=h\left(\frac{1+\sqrt{1-C^{2}(\rho)}}{2}\right)
$$

where

$$
h(x) \equiv-x \log _{2} x-(1-x) \log _{2}(1-x) .
$$

The quantity $C(\rho)$, referred to by Wootters as concurrence, is defined by

$$
C(\rho) \equiv \max \left\{0, \lambda_{m}-\lambda_{1}-\lambda_{2}-\lambda_{3}\right\},
$$

where $\lambda_{m}, \lambda_{1}, \lambda_{2}$, and $\lambda_{3}$ are the square roots of eigenvalues of the matrix $\rho \tilde{\rho}$, set in a decreasing order, with $\lambda_{m}$ being the maximum eigenvalue. The matrix $\tilde{\rho}$ denotes a spinflipped state:

$$
\tilde{\rho} \equiv\left(\sigma_{y} \otimes \sigma_{y}\right) \rho^{*}\left(\sigma_{y} \otimes \sigma_{y}\right) .
$$

The values of the entanglement of formation range from 0 to 1. Furthermore, $E_{F}$ is a monotonically increasing function of 
$C$. The values of the concurrence $C$, in turn, range from 0 to 1 , as the values of $E_{F}$ do. Consequently, the concurrence itself can be considered as a proper measure of the entanglement of formation. Note that the entanglement of formation is the only kind of entanglement studied in the present paper. For simplicity, we shall often refer to it simply as entanglement.

\section{A. On $\mathfrak{I}$, the working basis set for a system of two qubits}

The most general expression [22] of a mixed state $\rho$ of the space $S_{1 / 2}^{(1)} \otimes S_{1 / 2}^{(2)}$ is

$$
\begin{aligned}
\rho= & \frac{1}{16}\left\{1 \otimes 1+\left(\sum_{i=1}^{3} r_{i} \sigma_{i}\right) \otimes 1+1 \otimes\left(\sum_{i=1}^{3} s_{i} \sigma_{i}\right)\right. \\
& \left.+\sum_{i=1}^{3} \sum_{j=1}^{3} t_{i j} \sigma_{i} \otimes \sigma_{j}\right\},
\end{aligned}
$$

where $r_{i}, s_{i}$, and $t_{i j}$ are real parameters. Due to the excessive number of involved parameters, the direct use of this expression would make the calculation too complicated. For this reason we decided to limit our investigation to a set defined by

$$
\mathfrak{I} \equiv\left\{\rho: C(\rho)=2 P_{m}-1>0\right\},
$$

where $P_{m}$ denotes the greatest eigenvalue of the density matrix $\rho$ describing the quantum state. On behalf of future developments, we remark that the condition $P_{m}>1 / 2$ makes this maximum value unique. The adoption of the set $\mathfrak{I}$, as our working set, does not rule out the possibility of considering physical conditions of interest for the field of quantum teleportation. In fact, the set $\mathfrak{I}$ contains states with positive entanglement of formation $\left[E_{F}(\rho)>0\right]$ that are described by density matrices corresponding to the solution of the equation

$$
\rho \tilde{\rho}=\rho^{2} .
$$

These density matrices, recently used by Bennett et al. [21] (also see Ref. [23]), become diagonal when expressed in the Bell basis set. The set $\mathfrak{I}$ contains entangled Werner states [24] as well as maximally entangled states. We also note that any mixed state can be brought into a diagonal form in the Bell basis set by random bilateral rotations [21]. This implies that, in spite of the simplification made, our discussion is still rather general. The problem under discussion here is the significance of the Tsallis entropy as a measure of entanglement. The working set of states that we select, $\mathfrak{I}$, does not conflict with the possibility of discussing this issue with reference to one of the relevant physical conditions recently examined by investigators in this field of research $[21,23]$. In fact, we shall study the change of the Tsallis entropy indicator upon the entanglement change of a given pair of particles. This change might be the result of a purification process such as that studied by Bennett and co-workers [21,23]. These authors discussed the purification of a pair of Werner states, showing how to increase the entanglement of formation of one pair at the price of decreasing that of another. Here we discuss the problem of detecting this increased entanglement with the Tsallis entropy. We found it to be relatively easy to establish this important result within the set $\mathfrak{I}$. However, we cannot rule out the possibility that this property is shared by any other set of states, resulting in the concurrence being an increasing function of $P_{m}$. In all these cases we might find the same property of the entropy being proportional to the inverse of the entanglement.

It is worth anticipating an aspect of fundamental importance. The plausible reason why, as we shall see, the inverse of the Tsallis entropy indicator is a successful measure of entanglement, in the set $\mathfrak{I}$, is the following fundamental fact: The nonextensive nature of Tsallis entropy would make sense only in a world where it were impossible to create uncorrelated systems, and consequently, a vanishing entanglement. In fact, the Tsallis entropy is not the sum of the entropies of the parts, not even when these parts are uncorrelated. The condition that we set, of nonvanishing entanglement, as stated earlier, is equivalent to ruling out the occurrence of a splitting of the system into two uncorrelated parts. Therefore, it corresponds to an ideal condition for the application of the Tsallis entropy. We shall come back to this important issue in Sec. IV.

\section{B. Parametrization of the eigenvalues of the statistical density matrix}

Here we adopt a perspective of thermodynamic kind, inspired by the lines proposed by Plenio and Vedral [4]. We assume that a purification protocol yields an entanglement change $\Delta E_{F}$. We aim at establishing a change of the nonextensive entropy corresponding to the same "thermodynamic" transformation. To solve this delicate problem we imagine the statistical density matrix $\rho$, belonging to the set $\mathfrak{I}$, to be a function of a real parameter $\xi$, belonging to an interval $\left[\xi_{1}, \xi_{2}\right]$, which can be thought of as playing the same role as that of variables like pressure, temperature, and volume in the state transformations in ordinary thermodynamics. We assume that the initial and final conditions correspond to $\rho\left(\xi_{1}\right)$ and $\rho\left(\xi_{2}\right)$, respectively, so that $\Delta E_{F} \equiv E_{F}\left(\rho\left(\xi_{2}\right)\right)$ $-E_{F}\left(\rho\left(\xi_{1}\right)\right)$. We set the dependence of $\rho$ on $\xi$ in such a way as to fulfill the constraint imposed by the norm conservation $\operatorname{Tr} \rho=1$, and, among the infinitely large number of possibilities fitting these conditions, we select the form most convenient for the purpose of evaluating $\Delta S_{q} \equiv S_{q}\left(\rho\left(\xi_{2}\right)\right)$ $-S_{q}\left(\rho\left(\xi_{1}\right)\right)$. Let $P_{m}$ be the largest of the four eigenvalues of the statistical density matrix $\rho$, and let us denote the other three eigenvalues by $P_{1}, P_{2}$, and $P_{3}$. These eigenvalues are assumed to be functions of the parameter $\xi$ with the following condition: in the whole interval $\left[\xi_{1}, \xi_{2}\right]$, we have

$$
P_{m}(\xi)>\frac{1}{2} \text {. }
$$

The derivatives $d P_{m} / d \xi$ and $d P_{j} / d \xi$, with $j$ ranging from 1 to 3 , are assumed to be finite. The norm-conservation constraint enforces the condition

$$
\frac{d P_{m}}{d \xi}=-\frac{d P_{1}}{d \xi}-\frac{d P_{2}}{d \xi}-\frac{d P_{3}}{d \xi} .
$$


As pointed out earlier, concurrence is equivalent to the entanglement of formation and, in the set $\mathfrak{I}$, concurrence is a strictly increasing monotonic function of $P_{m}$. Thus the change of $P_{m}$ will be either positive or negative according to whether the entanglement change is positive or negative.

We shall assume that in the whole interval $\left[\xi_{1}, \xi_{2}\right]$ the derivative $d P_{m} / d \xi$ is either always positive or always negative. As stressed earlier, this convenient choice is legitimized by the fact that, as in ordinary thermodynamics, we are considering a state transformation, consequently both the entanglement and entropy changes depend only on the initial and final states, and are independent of the paths used to connect these two states.

\section{Failure of the von Neumann entropy}

Here we show that the von Neumann entropy, namely, the quantum version of the Shannon information, turns out to be inadequate as an entanglement indicator. This is so because, as we see, the von Neumann entropy can either increase or decrease, corresponding to the entanglement change $\Delta E_{F}$, regardless of whether the entanglement change is positive or negative. This conclusion agrees with the remarks in the recent work by Brukner and Zeilinger [9].

We note that to prove the inadequacy of the von Neumann entropy as an indicator of entanglement, it is enough to find a case where the sign of the entropy change is not strictly determined by that of the entanglement change. Thus let us consider the special physical condition corresponding to

$$
\frac{d P_{3}}{d \xi}=0
$$

in the whole interval $\left[\xi_{1}, \xi_{2}\right]$. The von Neumann entropy, corresponding to $q=1$, and consequently denoted as $S_{1}$, reads

$$
S_{1}(\rho)=-P_{m} \ln P_{m}-\sum_{j=1}^{3} P_{j} \ln P_{j},
$$

and its dependence upon the parameter $\xi$ is given by

$$
\frac{d S_{1}}{d \xi}=-\frac{d P_{m}}{d \xi} \ln \left(\frac{P_{m}}{P_{1}}\right)+\frac{d P_{2}}{d \xi} \ln \left(\frac{P_{2}}{P_{1}}\right) .
$$

Let us consider, for example, the case $d P_{m} / d \xi<0$. As noted earlier, in this case when the entanglement of the mixed state is a strictly decreasing function of $\xi$, the entanglement change $\Delta E_{F}$ must be negative. However, using Eqs. (15) and (14) we see that the sign of $d S_{1} / d \xi$ depends on the special values selected for the parameters $P_{m}, P_{1}, P_{2}$ and for the corresponding $\xi$ derivatives. Thus it is possible to realize either the inequality

$$
\frac{d P_{m}}{d \xi}<\frac{d P_{2}}{d \xi} \ln \left(\frac{P_{1}}{P_{2}}\right) / \ln \left(\frac{P_{m}}{P_{1}}\right),
$$

which would lead to an entropy increase, or the opposite inequality, which would yield an entropy decrease. This proves that the sign of the von Neumann entropy change is not correlated to that of the entanglement, and that consequently the von Neumann entropy is not an adequate entanglement indicator.

\section{Search of critical entropy indexes as a function of the $\xi$ transformation of the statistical density matrix eigenvalues}

The theoretical developments of this subsection show that the failure of the von Neumann entropy is a consequence of the fact that the von Neumann entropy means $q=1$, and this entropy index is smaller than, or equal to, a critical value $Q\left(\rho_{1}, \rho_{2}\right)$, where $\rho_{1}$ and $\rho_{2}$ denote initial and final states, respectively. In Sec. IIIE we shall show, in fact, that for $q$ $>Q\left(\rho_{1}, \rho_{2}\right)$ the nonextensive entropy becomes a monotonic function of the entanglement strength, inversely proportional to it: the condition $\Delta E_{F}>0$ yields $\Delta S_{q}<0$, while the condition $\Delta E_{F}<0$ yields $\Delta S_{q}>0$.

First, we show that the property that we plan to prove is a plausible property. For this purpose, let us study the $\xi$ derivative of the nonextensive entropy. This quantity reads

$$
\frac{d S_{q}}{d \xi}=-\frac{q P_{m}^{q-1}}{q-1} \frac{d P_{m}}{d \xi}\left\{1+\sum_{j=1}^{3}\left(\frac{d P_{m}}{d \xi}\right)^{-1}\left(\frac{P_{j}}{P_{m}}\right)^{q-1} \frac{d P_{j}}{d \xi}\right\} .
$$

We remind the reader that $P_{m}$ is the largest eigenvalue of $\rho$, thereby implying the property

$$
0 \leqslant \frac{P_{j}}{P_{m}}<1 .
$$

By using these inequalities and the assumption, made in Sec. III B, that the derivatives $d P_{j} / d \xi$ and $d P_{m} / d \xi$ are finite, we obtain

$$
\lim _{q \rightarrow+\infty}\left[1+\sum_{j=1}^{3}\left(\frac{P_{j}}{P_{m}}\right)^{q-1}\left(\frac{d P_{m}}{d \xi}\right)^{-1}\left(\frac{d P_{j}}{d \xi}\right)\right]=1 .
$$

This simple result implies that great enough values of the entropy index yield the important property

$$
\operatorname{sgn}\left(\frac{d E_{F}}{d \xi}\right)=\operatorname{sgn}\left(\frac{d P_{m}}{d \xi}\right)=-\operatorname{sgn}\left(\frac{d S_{q}}{d \xi}\right),
$$

which, in turn, means that increasing entanglement in the $\xi$ transformation yields a decreasing entropy, and vice versa.

After proving the monotonic dependence of the nonextensive entropy on the entanglement strength in the asymptotic limit, we now illustrate a recipe necessary to determine the critical entropic index, namely, the value of $q$ beyond which the monotonic dependence of entropy on entanglement is insured. More precisely, we provide the recipe for two critical indexes, rather than one, according to whether we consider the case of entanglement decrease [case (a)] or increase [case (b)]. These two critical entropic indexes are denoted by $Q^{\star}\left(\xi_{1}, \xi_{2}\right)$ and $Q^{\star \star}\left(\xi_{1}, \xi_{2}\right)$, respectively. This means that $\Delta S_{q}>0$ if $\Delta E<0$, and $\Delta S_{q}<0$ if $\Delta E>0$, provided that $q$ $>Q^{\star}\left(\xi_{1}, \xi_{2}\right)$ and $q>Q^{\star \star}\left(\xi_{1}, \xi_{2}\right)$, respectively. 
The critical values $Q^{\star}\left(\xi_{1}, \xi_{2}\right)$ and $Q^{\star \star}\left(\xi_{1}, \xi_{2}\right)$ are defined as follows:

$$
\begin{aligned}
Q^{\star}\left(\xi_{1}, \xi_{2}\right) & \equiv \sup _{\xi \in\left[\xi_{1}, \xi_{2}\right]}\left\{q^{\star}(\xi)\right\}, \\
Q^{\star \star}\left(\xi_{1}, \xi_{2}\right) & \equiv \sup _{\xi \in\left[\xi_{1}, \xi_{2}\right]}\left\{q^{\star \star}(\xi)\right\} .
\end{aligned}
$$

The auxiliary functions $q^{\star}(\xi)$ and $q^{\star \star}(\xi)$ are defined by

$$
\begin{gathered}
q^{\star}(\xi) \equiv \max \left\{1, \alpha_{1}^{\star}(\xi), \alpha_{2}^{\star}(\xi), \alpha_{3}^{\star}(\xi)\right\}, \\
q^{\star \star}(\xi) \equiv \max \left\{1, \alpha_{1}^{\star \star}(\xi), \alpha_{2}^{\star \star}(\xi), \alpha_{3}^{\star \star}(\xi)\right\} .
\end{gathered}
$$

The functions $\alpha_{j}^{\star}(\xi)$, with the subscript $j$ running from 1 to 3 , are functions of the interval $\left[\xi_{1}, \xi_{2}\right]$ given by

$$
\alpha_{j}^{\star}(\xi) \equiv 1+\left[\ln \left(\frac{P_{m}}{P_{j}}\right)\right]^{-1} \ln \left(3\left|\frac{d P_{m}}{d \xi}\right|^{-1} \frac{d P_{j}}{d \xi}\right)
$$

if the conditions $P_{j}(\xi)>0$ and $d P_{j} / d \xi>0$ apply. If these conditions do not apply, we set $\alpha_{j}^{\star}(\xi)=1$. The functions $\alpha_{j}^{\star \star}(\xi)$ are given by

$$
\alpha_{j}^{\star \star}(\xi) \equiv 1+\left[\ln \left(\frac{P_{m}}{P_{j}}\right)\right]^{-1} \ln \left[3\left(\frac{d P_{m}}{d \xi}\right)^{-1}\left|\frac{d P_{j}}{d \xi}\right|\right]
$$

if the conditions $P_{j}(\xi)>0$ and $d P_{j} / d \xi<0$ apply. If these conditions do not apply, we set $\alpha_{j}^{\star \star}(\xi)=1$. The proof of this important recipe is given in the Appendix. Note that we have not discussed the problem of the possible divergence of $Q^{\star}\left(\xi_{1}, \xi_{2}\right)$ or $Q^{\star \star}\left(\xi_{1}, \xi_{2}\right)$. We shall come back to this issue in Sec. IIIE, where we shall consider, without losing any generality, a special parametrization of the eigenvalues within which, as we shall see, the critical index $Q\left(\rho_{1}, \rho_{2}\right)$ will be proved to be finite.

\section{E. Search of a critical entropy index as a function of initial and final states}

Now let us see how to use the earlier results to make predictions in the case where the transformation and the ensuing entanglement change are described only by the initial and final states $\rho_{1}$ and $\rho_{2}$, with the density matrices belonging to the set $\mathfrak{I}$ defined by Eq. (11). The main idea is to build up auxiliary states $\rho_{B}^{(1)}$ and $\rho_{B}^{(2)}$, equivalent to $\rho_{1}$ and $\rho_{2}$, respectively, as far as their entanglement and entropy are concerned, but fulfilling the condition of being connected the one to the other by one of the $\xi$ transformations described in Sec. IIIC. This makes these states compatible with earlier prescriptions, and thus with earlier results. The states $\rho_{B}^{(1)}$ and $\rho_{B}^{(2)}$ are defined as follows. Let $P_{m}^{(1)}, P_{1}^{(1)}, P_{2}^{(1)}$, and $P_{3}^{(1)}$ denote the eigenvalues of the density matrix $\rho_{1}$, while $P_{m}^{(2)}, P_{1}^{(2)}, P_{2}^{(2)}$, and $P_{3}^{(2)}$ denote the eigenvalues of the density matrix $\rho_{2}$; we define the auxiliary states $\rho_{B}^{(1)}$ and $\rho_{B}^{(2)}$ by the expressions

$$
\rho_{B}^{(1)} \equiv P_{m}^{(1)}\left|e_{m}\right\rangle\left\langle e_{m}\left|+\sum_{j=1}^{3} P_{j}^{(1)}\right| e_{j}\right\rangle\left\langle e_{j}\right|
$$

and

$$
\rho_{B}^{(2)} \equiv P_{m}^{(2)}\left|e_{m}\right\rangle\left\langle e_{m}\left|+\sum_{j=1}^{3} P_{j}^{(2)}\right| e_{j}\right\rangle\left\langle e_{j}\right|
$$

where the set $\left\{\left|e_{m}\right\rangle,\left|e_{j}\right\rangle, j=1,2,3\right\}$ is the Bell basis set [21], no matter what the order is. It is easy to check that these quantum states have the following properties: (i) they belong to the set $\mathfrak{I}$, (ii) $E_{F}\left(\rho_{1}\right)=E_{F}\left(\rho_{B}^{(1)}\right)$ and $E_{F}\left(\rho_{2}\right)=E_{F}\left(\rho_{B}^{(2)}\right)$, and (iii) $S_{q}\left(\rho_{1}\right)=S_{q}\left(\rho_{B}^{(1)}\right)$ and $S_{q}\left(\rho_{2}\right)=S_{q}\left(\rho_{B}^{(2)}\right)$.

Now let us introduce the transformation $\Xi_{\xi}\left[\rho_{B}^{(1)}, \rho_{B}^{(2)}\right]$, defined by

$$
\Xi_{\xi}\left[\rho_{B}^{(1)}, \rho_{B}^{(2)}\right]\left(\rho_{B}^{(1)}\right) \equiv P_{m}(\xi)\left|e_{m}\right\rangle\left\langle e_{m}\left|+\sum_{j=1}^{3} P_{j}(\xi)\right| e_{j}\right\rangle\left\langle e_{j}\right|,
$$

where the $\xi$ evolutions of $P_{m}(\xi)$ and $P_{j}(\xi)$ are given by

$$
P_{m}(\xi) \equiv P_{m}^{(1)}+\xi\left(P_{m}^{(2)}-P_{m}^{(1)}\right)
$$

and

$$
P_{j}(\xi) \equiv P_{j}^{(1)}+\xi\left(P_{j}^{(2)}-P_{j}^{(1)}\right),
$$

with $j$ running from 1 to 3 , respectively, and $\xi$ belonging to the interval $[0,1]$. The transformation $\Xi_{\xi}$ has the required properties: (a) it keeps the state $\Xi_{\xi}\left[\rho_{B}^{(1)}, \rho_{B}^{(2)}\right]\left(\rho_{B}^{(1)}\right)$ within the set $\mathfrak{I}$ for every value of $\xi$ belonging to the interval $[0,1]$, (b) $\Xi_{0}\left[\rho_{B}^{(1)}, \rho_{B}^{(2)}\right]\left(\rho_{B}^{(1)}\right)=\rho_{B}^{(1)}$, and (c) $\Xi_{1}\left[\rho_{B}^{(1)}, \rho_{B}^{(2)}\right]\left(\rho_{B}^{(1)}\right)$ $=\rho_{B}^{(2)}$. Note that the functions $P_{m}(\xi), P_{1}(\xi), P_{2}(\xi)$, and $P_{3}(\xi)$ are eigenvalues of the quantum states $\Xi_{\xi}\left[\rho_{B}^{(1)}\right.$, $\left.\rho_{B}^{(2)}\right]\left(\rho_{B}^{(1)}\right)$, and are defined in the interval $[0,1]$. They fulfill the properties of Eq. (13), the parameter conditions of Sec. III B, the relation $d P_{m} / d \xi>0$ in the case $P_{m}^{(1)}<P_{m}^{(2)}$, and the relation $d P_{m} / d \xi<0$ in the case $P_{m}^{(1)}>P_{m}^{(2)}$. This makes it possible for us to use $Q^{\star}\left(\xi_{1}, \xi_{2}\right)$ of Eq. (23) and $Q^{\star \star}\left(\xi_{1}, \xi_{2}\right)$ of Eq. (24), and the relations on which these quantities rest as well, to derive $Q\left(\rho_{1}, \rho_{2}\right)$. This is done as follows. We write the explicit forms that $Q^{\star}\left(\xi_{1}, \xi_{2}\right)$ and $Q^{\star \star}\left(\xi_{1}, \xi_{2}\right)$ gain when $\xi_{1}=0$ and $\xi_{2}=1$. Applying the transformations of Eqs. (32) and (33) to the prescriptions of Eqs. (23)-(28) , we obtain the following expressions:

$$
Q^{\star}(0,1) \equiv \sup _{\xi \in[0,1]} \max \left\{1, \beta_{1}^{\star}(\xi), \beta_{2}^{\star}(\xi), \beta_{3}^{\star}(\xi)\right\} .
$$

Here the function $\beta_{j}^{\star}(\xi)$, with $j=1,2$, and 3, is defined as follows. If the constraints

$$
P_{j}^{(2)}(\xi)>P_{j}^{(1)}(\xi) \text { and } P_{j}^{(1)}+\xi\left(P_{j}^{(2)}-P_{j}^{(1)}\right)>0,
$$

with $j=1,3$, hold true, we set

$$
\beta_{j}^{\star}(\xi) \equiv 1+\frac{\ln \left(3 \frac{P_{j}^{(1)}-P_{j}^{(2)}}{P_{m}^{(2)}-P_{m}^{(1)}}\right)}{\ln \left(\frac{P_{m}^{(1)}+\xi\left(P_{m}^{(2)}-P_{m}^{(1)}\right)}{P_{j}^{(1)}+\xi\left(P_{j}^{(2)}-P_{j}^{(1)}\right)}\right)} .
$$


If the constraints of Eq. (35) do not apply, we set $\beta_{j}^{\star}(\xi)$ $=1$. It is evident that the functions $\beta_{j}^{\star}(\xi)$ are the counterparts of $\alpha_{j}^{\star}(\xi)$ in the particular case of the $\xi$ transformation described by Eqs. (32) and (33).

Note that this mathematical definition must be interpreted as follows. First we consider a given value of $\xi$ belonging to the interval $[0,1]$. Then we make the index $j$ run from 1 to 3 , we select indexes $j$ fulfilling the conditions $P_{j}^{(2)}(\xi)$ $>P_{j}^{(1)}(\xi)$ and $P_{j}^{(1)}+\xi\left(P_{j}^{(2)}-P_{j}^{(1)}\right)>0$, and calculate $\beta_{j}^{\star}(\xi)$ using the above definitions. Finally, we take the maximum of the values of a set whose components are given by $\beta_{j}^{\star}(\xi)$ and by 1 , and we make $\xi$ explore all possible values of the interval $[0,1]$. Thus we obtain an infinite set of maxima, from which we select the supremum. The resulting number defines the critical index of the left hand side of Eq. (34).

The resulting critical index is finite. To prove this important property we proceed as follows. We note that the term that could make $Q^{\star}(0,1)$ diverge is

$$
\frac{P_{m}^{(1)}+\xi\left(P_{m}^{(2)}-P_{m}^{(1)}\right)}{P_{j}^{(1)}+\xi\left(P_{j}^{(2)}-P_{j}^{(1)}\right)} .
$$

We denote this term by $\gamma(\xi)$. The special condition resulting in the divergence of the critical index would be given by $\gamma$ $\rightarrow 1^{+}$. We observe that $\gamma(\xi)$ is either an increasing (decreasing) or constant function of $\xi$ depending on whether the quantity $P_{j}^{(1)} P_{m}^{(2)}-P_{m}^{(1)} P_{j}^{(2)}$ is positive (negative) or equal to 0 . So the minimum value of $\gamma(\xi)$ is $\gamma(0)=P_{m}^{(1)} / P_{j}^{(1)}$ in the case of $d \gamma / d \xi>0$, and $\gamma(1)=P_{m}^{(2)} / P_{j}^{(2)}$ in the case of $d \gamma / d \xi<0$. In the remaining case $d \gamma / d \xi=0$, the two minima obtain the same value. From these properties we obtain the inequality

$$
\begin{aligned}
Q^{\star}(0,1) \leqslant & \max _{j=1,2,3}\left\{1+\left[\ln \left(\min _{j=1,2,3}\left\{\frac{P_{m}^{(1)}}{P_{j}^{(1)}}, \frac{P_{m}^{(2)}}{P_{j}^{(2)}}\right\}\right)\right]^{-1}\right. \\
& \left.\times\left|\ln \left(3\left|\frac{P_{j}^{(2)}-P_{j}^{(1)}}{P_{m}^{(1)}-P_{m}^{(2)}}\right|\right)\right|\right\},
\end{aligned}
$$

proving that $Q^{\star}(0,1)$ is finite.

As for $Q^{\star \star}(0,1)$, we obtain

$$
Q^{\star \star}(0,1) \equiv \sup _{\xi \in[0,1]} \max \left\{1, \beta_{1}^{\star \star}(\xi), \beta_{2}^{\star \star}(\xi), \beta_{3}^{\star \star}(\xi)\right\} .
$$

If the constraints

$$
P_{j}^{(1)}(\xi)>P_{j}^{(2)}(\xi) \quad \text { and } \quad P_{j}^{(1)}+\xi\left(P_{j}^{(2)}-P_{j}^{(1)}\right)>0
$$

hold true, we set

$$
\beta_{j}^{\star \star}(\xi) \equiv 1+\frac{\ln \left(3 \frac{P_{j}^{(1)}-P_{j}^{(2)}}{P_{m}^{(2)}-P_{m}^{(1)}}\right)}{\ln \left(\frac{P_{m}^{(1)}+\xi\left(P_{m}^{(2)}-P_{m}^{(1)}\right)}{P_{j}^{(1)}+\xi\left(P_{j}^{(2)}-P_{j}^{(1)}\right)}\right)} .
$$

If the constraints of Eq. (39) do not apply, we set $\beta_{j}^{\star \star}(\xi)$ $=1$.

The criterion adopted to define this critical index is the same as that illustrated earlier to properly define the critical index of Eq. (34). Thus we can prove that $Q^{\star \star}(0,1)$ is finite, adopting a procedure analogous to that used for $Q^{\star}(0,1)$. In this case we arrive at the inequality

$$
\begin{aligned}
Q^{\star \star}(0,1) \leqslant & \max _{j=1,2,3}\left\{1+\left[\ln \left(\min _{j=1,2,3}\left\{\frac{P_{m}^{(1)}}{P_{j}^{(1)}}, \frac{P_{m}^{(2)}}{P_{j}^{(2)}}\right\}\right)\right]^{-1}\right. \\
& \left.\times\left|\ln \left(3\left|\frac{P_{j}^{(1)}-P_{j}^{(2)}}{P_{m}^{(2)}-P_{m}^{(1)}}\right|\right)\right|\right\},
\end{aligned}
$$

which shows in fact that also $Q^{\star \star}(0,1)$ is finite.

At this stage we can finally define the critical value $Q\left(\rho_{1}, \rho_{2}\right)$. This is given by

$$
Q\left(\rho_{1}, \rho_{2}\right) \equiv \max \left\{Q^{\star}(0,1), Q^{\star \star}(0,1)\right\} .
$$

On the basis of the theoretical treatment described earlier, we conclude that $Q\left(\rho_{1}, \rho_{2}\right)$ is finite and that for any initial and final states $\rho_{1}$ and $\rho_{2}$, respectively, belonging to the set $\mathfrak{I}$, with different entanglement, $E_{F}\left(\rho_{1}\right) \neq E_{F}\left(\rho_{2}\right)$, the corresponding entropy change $\Delta S_{q}$ is positive or negative, according to whether $\Delta E<0$ or $\Delta E>0$. Note that we found $+\infty$ $>Q\left(\rho_{1}, \rho_{2}\right) \geqslant 1$. As a consequence of the pseudoadditivity of Eq. (2), the adoption of a value of the entropy index larger than the unity makes the entropy of the whole system smaller than the sum of the entropies of the two parts. However, in this paper we never make a direct use of this property, since, as stressed earlier, our treatment is valid only in the case of nonvanishing entanglement, which rules out the possibility of realizing the factorized condition behind Eq. (2).

\section{F. From the nonextensive entropy to the entanglement of formation}

As a purpose of this subsection, we try to prove a property that is the reverse of that discussed in Sec. III E. Ideally, the reverse of the property of Sec. IIIE should be expressed as follows. Let us focus our attention on a transformation from an initial state $\rho_{1}$ to a final state $\rho_{2}$, both belonging to the set $\Im$. Let us consider a case where this transformation causes the nonextensive entropy $S_{q}$ to increase (decrease). Then the entanglement decreases (increases) if an entropy index $q$ larger than the critical value $Q$ is adopted. Unfortunately, we cannot prove this property in this attractive form, but only under weaker conditions. This is so because a transformation resulting in an entropy change does note necessarily imply an entanglement change. We note that the entanglement, expressed in the set $\mathfrak{I}$, is a function of the eigenvalue $P_{m}$ only, while the nonextensive entropy is a function of all four eigenvalues. Thus the entropy can change without implying a corresponding entanglement change. The same difficulty is shared by the nonextensive entropy. However, upon an increase of the entropy index $q$ the dependence of the nonextensive entropy on the other three eigenvalues becomes weaker and weaker. In the case of enough great values of the 
entropy index $q$ the nonextensive entropy becomes virtually independent of the other three eigenvalues. This is the reason why in Sec. III E we could find a way to make the nonextensive entropy become a monotonic function of the entanglement. We want to remark that in general the entropy critical index is not the same as that used in Sec. IIIE.

We believe that one of the benefits resulting from the adoption of the set $\mathfrak{I}$, and of very large entropy indices as well, is that the margin of entanglement dependence on the entropy is significantly reduced. Nevertheless, we are forced to make a weaker request for the reverse of the property discussed in Sec. IIIE. We shall show, in fact, that if the entropy increases (decreases), and the entanglement changes, then the entanglement decreases (increases), for entropy indices $q$ larger than a critical value $Q^{(S)}$, not necessarily equal to $Q$. The conditions emphasized by the adoption of italics make the property weaker than we would wish. Even in this case we have to assume the entropy index to be larger than a critical value. We denote this critical value with the symbol $Q^{(S)}$ because, as mentioned earlier, we cannot prove that it is identical to the critical entropy index $Q$ of an Sec. IIIE.

In the case of an entropy increase, by expressing the nonextensive entropy as a function of its four eigenvalues, we obtain

$$
\left(\frac{P_{m}^{(2)}}{P_{m}^{(1)}}\right)^{q}<1+\sum_{j=1}^{3}\left[\left(\frac{P_{j}^{(1)}}{P_{m}^{(1)}}\right)^{q}-\left(\frac{P_{j}^{(2)}}{P_{m}^{(1)}}\right)^{q}\right]
$$

Since the two eigenstates have different entanglements, we have $P_{m}^{(2)} / P_{m}^{(1)} \neq 1$. The inequality of Eq. (43) must hold true in the case of entropy indices arbitrarily larger than $Q^{(S)}$, and consequently must hold true also for values much larger than the unity. As a consequence we reach the conclusion that $P_{m}^{(2)}<P_{m}^{(1)}$, an inequality that in the set $\mathfrak{I}$ is equivalent to $\Delta E_{F}<0$. The opposite conclusion would be reached in the case of a negative $\Delta S_{q}$.

In spite of earlier restrictions, we can use the obtained results to illustrate one of the most interesting findings of this work. This is as follows. Let us consider a generic subset $\mathfrak{I}^{\prime}$, of set $\mathfrak{I}$, fulfilling only the request of containing a finite number of states, with different entanglements. Then we can conclude that these entanglements are equivalent to the inverse of the nonextensive entropy, provided that entropy indices $q$ are larger than a given value $Q_{\mathfrak{J}^{\prime}}$, which is given by the following formula:

$$
Q_{\mathfrak{I}^{\prime}} \equiv \max \left\{Q\left(\rho_{i}, \rho_{j}\right), \forall \rho_{i}, \rho_{j} \in \mathfrak{I}^{\prime}, i \neq j\right\}
$$

In the set $\mathfrak{I}^{\prime}$ for entropy indices larger than the critical value the ordering in the direction of increasing (decreasing) entanglement is equivalent to ordering in the direction of decreasing (increasing) entropy. A significant consequence of this is that entropy minimization yields a maximally entangled state and entropy maximization yields a minimally entangled state.

An attractive, albeit heuristic way, of illustrating the same conclusions is given by the formula

$$
\begin{aligned}
E_{q}^{(e f f)}(\rho) \equiv & \frac{2}{\pi} \arctan \left\{\sum_{i=1}^{4} \Theta\left(P_{i}-\sum_{k \neq i} P_{k}\right)\right. \\
& \left.\times\left(3-4 S_{2}(\rho)-4 \sum_{k \neq i} P_{k}^{2}\right) S_{q}^{-1}\right\},
\end{aligned}
$$

which establishes a direct connection between entanglement and entropy. The quantity $E_{q}^{(e f f)}$ is "equivalent" to the entanglement, in the sense that it increases or decreases upon an increase or decrease of the entanglement strength. Furthermore, it is equal to 1 when the entanglement is 1 , and tends to vanish with the entanglement measure tending to zero. The key ingredient of this heuristic formula is the term arctan, and the factor $\sum_{i=1}^{4} \Theta\left(P_{i}-\Sigma_{k \neq i} P_{k}\right)\left(3-4 S_{2}(\rho)\right.$ $-4 \Sigma_{k \neq i} P_{k}^{2}$ ). Without arctan, the condition $P_{m} \rightarrow 1^{-}$would generate divergencies. Furthermore with $P_{m} \rightarrow(1 / 2)^{+}$the inverse of the entropy would tend to a minimum which would be different from 0 , which is the right value. With the factor $\sum_{i=1}^{4} \Theta\left(P_{i}-\sum_{k \neq i} P_{k}\right)\left[3-4 S_{2}(\rho)-4 \sum_{k \neq i} P_{k}^{2}\right]$, we dispose of the divergencies and we succeed in ensuring that the quantity $E_{q}^{(e f f)}$ tends to vanish with the entanglement tending to zero. Note that this ad hoc factor is nothing but the square of the concurrence. In principle, one could express the concurrence in terms of $S_{2}$, but this would not afford the attractive condition of the entanglement being a monotonically increasing function of the inverse of the nonextensive entropy.

In conclusion, we find that entanglement increase implies entropy decrease, and vice versa. This property must be compared with the results of the work of Abe and Rajagopal [15]. These authors adopted the principle of entropy maximization under suitable constraints to infer a plausible form of physical state, and concluded that the entangled states are the important result of this maximization process. Here we adopt a different perspective, based on the fact that the definition of entanglement of formation is already inspired to statistical mechanics [20]. Within this perspective the state of maximum entanglement corresponds to the minimum amount of information necessary to describe the state. Within this same perspective, the amount of information necessary to describe the state becomes increasingly larger upon reducing the entanglement strength. From an intuitive point of view, the occurrence of decoherence, is judged by many authors [25] to be the key condition to derive classical from quantum physics, implies a significant entropy increase. However, decoherence, as a form of real wave-function collapse [26], implies the breakdown, in the long-time limit, of the entanglement condition, and, as a consequence, the breakdown of the theory itself of the present paper. The result of this paper has to be considered within this perspective. As it appears from the literature on this new and exciting subject, the thermodynamic significance of the processes of quantum teleportation is a very delicate and difficult issue. We are inclined to believe that the adoption of a nonextensive form of entropy might be of some relevance, under specific restrictions. The first is that real wave-function collapses are ignored, and the second is that, in a world dominated by quantum entanglement, the condition of maximum entanglement is perceived 
as that requiring the minimum amount of information. In other words, increasing entanglement means smaller, rather than larger, entropy values.

\section{TSALLIS ENTROPY AT WORK: DEPHASING PROCESSES IN THE BELL BASIS SET}

Before ending this paper, it is convenient to illustrate another interesting result that does not require a restriction to the set $\mathfrak{I}$. This has to do with an important result obtained by Bennett et al. [21]. These authors studied entanglement changes as a function of a dephasing process. More precisely, they focused their attention on the transformation

$$
D_{B}=\frac{1}{4} \sum_{i=0}^{3} U_{i}^{\dagger} \rho U_{i},
$$

which brings the initial condition described by the density matrix $\rho$, expressed in the Bell basis, into the diagonal form

$$
\left[D_{B}\right]_{i j} \equiv \delta_{i j}[\rho]_{i j},
$$

where the operators $U_{i}, i=0,1,2,3$ are $I, B_{x} B_{x}, B_{y} B_{y}$, and $B_{z} B_{z}$, respectively, and $B_{i}$ is the bilateral rotation of $\pi / 2$ around the $i$ th axis of the space $S_{1 / 2}^{(1)} \times S_{1 / 2}^{(2)}$. This bilateral rotation was defined by these authors [21] as

$$
B_{i}=\frac{1}{2}\left(I_{2 \times 2}-i \sigma_{1 i}\right)\left(I_{2 \times 2}-i \sigma_{2 i}\right) .
$$

Note that the matrix $D_{B}$ of Eq. (47) is the "diagonal" of the statistical density matrix $\rho$ expressed in the Bell basis, and that it results from a random application of four local unitary transformations, so that moving from the initial state $\rho^{(1)}$ to the state described by $D_{B}^{(1)}$ the entanglement cannot increase [27]. Consequently, we have

$$
E_{F}\left(\rho^{(1)}\right) \geqslant E_{F}\left(D_{B}^{(1)}\right) .
$$

We shall analyze these theoretical results by means of nonextensive entropy. The first analysis is made by focusing our attention on the natural values $n>1$ of the entropy index $q$. In this special case the nonextensive entropy reads as follows:

$$
S_{n}(\rho)=\frac{1-\operatorname{Tr}\left(U \rho U^{\dagger}\right)^{n}}{n-1}=\operatorname{Tr} \frac{\rho-\rho^{n}}{n-1} .
$$

Let us define the auxiliary function

$$
g_{n}(x) \equiv \frac{x-x^{n}}{n-1} .
$$

We note that this is a concave function. On the other hand, several years ago Wehrl [28] note this in that case we can write

$$
\operatorname{Tr} g_{n}\left(D_{B}\right) \geqslant \operatorname{Tr} g_{n}(\rho) .
$$

We note that $S_{n}(\rho)=\operatorname{Tr} g_{n}(\rho)$ and $S_{n}\left(D_{B}\right)=\operatorname{Tr} g_{n}\left(D_{B}\right)$. Consequently, we can write

$$
S_{n}\left(D_{B}\right) \geqslant S_{n}(\rho)
$$

The dephasing process makes it possible for us to generalize the results of Sec. III. Let us consider a transformation from an initial state described by a generic density matrix. As to the final state, we set the condition that it belongs to the set $\mathfrak{I}$. Let $p_{m}^{(1)}$ be the maximum of the diagonal elements of the initial state $\rho^{(1)}$, expressed in the Bell basis set. Let us also suppose that $p_{m}^{(1)}$ is larger than the maximum eigenvalue of the density matrix $\rho^{(2)}$, referring to the final state. This condition is expressed by the relation

$$
p_{m}^{(1)} \equiv \max _{i=1,2,3,4}\left[\rho^{(1)}\right]_{i i}>P_{m}^{(2)}>\frac{1}{2} .
$$

As a consequence of this relation, we have

$$
E_{F}\left(\rho^{(1)}\right) \geqslant E_{F}\left(D_{B}^{(1)}\right)>E_{F}\left(\rho^{(2)}\right)>0
$$

This is a transformation with a decreasing entanglement. On the basis of the results of Sec. III and of Eq. (53), we are in a position to find values of the entropy index $q$ such that the nonextensive entropy of the final state is larger than that of the initial state. This is done as follows. We move from the initial condition $\rho^{(1)}$ to $D_{B}^{(1)}$, through the dephasing process earlier described. As we have seen, with the adoption of natural values, larger than the unity, for the entropy indices, the entropy does not decrease. This means that

$$
S_{n}\left(D_{B}^{(1)}\right) \geqslant S_{n}\left(\rho^{(1)}\right)
$$

According to our assumptions, $D_{B}^{(1)}$ and $\rho^{(2)}$ belong to the set $\mathfrak{I}$. Thus we know, on the basis of the results of Sec. III, that there exists a critical value of the entropy index, $Q\left(D_{B}^{(1)}, \rho^{(2)}\right)$, beyond which the nonextensive entropy increases. If we choose critical values of the entropy index that are natural numbers larger than

$$
N \equiv\left[Q\left(D_{B}^{(1)}, \rho^{(2)}\right)\right],
$$

we conclude that the nonextensive entropy increases. As earlier anticipated, this has the effect of making more general the results of Sec. III.

\section{CONCLUSIONS}

This paper shows that in the set $\mathfrak{I}$, enforcing the important condition of a nonvanishing entanglement, the Tsallis entropy is a monotonic and decreasing function of the increasing entanglement. The entanglement is, in turn, a monotonic and decreasing function of the increasing entropy under the key restriction of transformations yielding an entanglement change. This conclusion was reached adopting a perspective taking the warning of a recent paper by Horodecki et al. [27] into account. As a matter of fact, these authors showed that the principle of entropy maximization yields fake entanglement, and consequently becomes questionable. We share the conviction of these authors and adopt in fact an approach that does not rest on the Jaynes principle [18,19]. Thus we establish a comparison between entanglement and nonexten- 
sive entropy without invoking the Jaynes principle. We do not need to maximize entropy after minimizing entanglement, as in Ref. [27], and the monotonic dependence of entropy on entanglement is a natural consequence of the adoption of suitably large entropy indices.

This means that we share the view of Rajagopal and Abe [15] that a nonextensive form of entropy can prove to be a convenient tool to study quantum teleportation. In this sense, this paper contributes to deepening our understanding of the significance of the Tsallis entropy. This entropy indicator does not split into the sum of two independent contributions, when applied to a system consisting of two uncorrelated subsystems. This suggests that this kind of entropy might be a proper theoretical tool only when applied to cases where a repartition into two uncorrelated systems is impossible. Quantum-mechanical systems, in principle, are significant examples where this condition applies, if environmental decoherence, or other kind of decoherence processes, is ignored. In this condition the Tsallis entropy, according to the main result of this paper, seems to work properly, provided that the warning of Ref. [27] is taken into account. This is where our procedure departs from the point of view of Rajagopal and Abe [15]. Their approach was still based on the Jaynes principle, supplemented by the choice of a suitable additional constraint, concerning the fluctuations around the average, as well as the ordinary constraint on the mean value (also see Ref. [29]). This procedure yields convincing, although nongeneral conclusions. Our approach, which unfortunately shares the lack of generality of Ref. [15], is based on a different perspective, aiming at identifying the inverse of entanglement with the non-extensive entropy.

We think the alternative perspective adopted in the present paper might contribute, as Refs. [15,29] do, to a better understanding of the thermodynamic nature of entanglement. We are afraid that the nonextensive entropy might become inefficient when we leave the physical condition where the no-cloning theorem and the principle of no-increasing entanglement, recently found by Horodecki and Horodecki [30], is broken. According to these authors the occurrence of real wave-function collapses, incompatible with the restriction of adopting unitary transformations, provokes a breakdown of this equivalence. In our opinion, the occurrence of real wave-function collapses is incompatible with the restriction of working on the set $\mathfrak{I}$, which enforces the condition of a nonvanishing entanglement. Thus we expect that in this case the theory of this paper, and with it the nonextensive entropy, does not work. To explore the uncertain border between quantum and classical mechanics we probably need to adopt a still more advanced perspective.

\section{APPENDIX}

This appendix is devoted to proving the crucial properties of the critical values defined by Eqs. (23)-(28). Let us consider case (a) first. As a consequence of $d E_{F} / d \xi<0$, from the limit of Eq. (21) we naturally obtain that an auxiliary function, $q^{\star}(\xi)$ in Eq. (25), exists such that $q>q^{\star}(\xi)$ yields

$$
1+\sum_{j=1}^{3}\left(\frac{d P_{m}}{d \xi}\right)^{-1}\left(\frac{P_{j}}{P_{m}}\right)^{q-1}\left(\frac{d P_{j}}{d \xi}\right)>0
$$

This critical value $q^{\star}(\xi)$ fulfilling the condition of Eq. (A1) is also not unique. We therefore adopt a criterion to estimate one of the possible critical values. This will imply that the resulting $Q\left(\rho_{1}, \rho_{2}\right)$ is not unique, but, as shown below, we shall be able to find at least one of the values fulfilling the earlier mentioned properties of $Q\left(\rho_{1}, \rho_{2}\right)$. The choice that we adopt to find one of the possible $q^{\star}(\xi)$ 's is as follows. We set the inequality

$$
\left|\frac{d P_{m}}{d \xi}\right|^{-1}\left(\frac{P_{j}}{P_{m}}\right)^{q-1} \frac{d P_{j}}{d \xi}<\frac{1}{3}
$$

for every value of the subscript $j$ running from 1 to 3 . We assume that this property holds true for any $q>q^{\star}(\xi)$. This set of conditions, after easy algebra, yields

$$
q^{\star}(\xi)=\max \left\{1, \alpha_{1}^{\star}(\xi), \alpha_{2}^{\star}(\xi), \alpha_{3}^{\star}(\xi)\right\} .
$$

As for the definition of $\alpha_{j}^{\star}(\xi)$, with the subscript $j$ running from 1 to 3 , we must distinguish two cases. The first is the case when the constraints

$$
P_{j}(\xi)>0 \quad \text { and } \quad d P_{j} / d \xi>0
$$

hold true. In this case, we set

$$
\alpha_{j}^{\star}(\xi) \equiv 1+\left[\ln \left(\frac{P_{m}}{P_{j}}\right)\right]^{-1} \ln \left(3\left|\frac{d P_{m}}{d \xi}\right|^{-1} \frac{d P_{j}}{d \xi}\right) .
$$

If the constraints of Eq. (A4) do not apply, i.e., either $P_{j}(\xi)=0$ or $d P_{j} / d \xi \leqslant 0$ applies, we set $\alpha_{j}^{\star}(\xi)=1$, in accordance with the inequality (A2) which is true for every value of the entropic index $q$ different from unity. The conclusion of this procedure is that we built up the auxiliary function $q^{\star}(\xi)$ in such a way that for any $q>q^{\star}(\xi)$ the condition

$$
\frac{d}{d \xi} E_{F}(\xi)<0
$$

yields

$$
\frac{d}{d \xi} S_{q}(\xi)>0
$$

On the basis of this result, the function $Q^{\star}\left(\xi_{1}, \xi_{2}\right)$, defined in Eq. (23),

$$
Q^{\star}\left(\xi_{1}, \xi_{2}\right) \equiv \sup _{\xi \in\left[\xi_{1}, \xi_{2}\right]}\left\{q^{\star}(\xi)\right\}
$$

has the properties described in Sec. III D. In fact, using Eq. (A6) and the ensuing inequality for $d S_{q} / d \xi$, we immediately conclude that for any $q$ fulfilling the inequality $q$ $>Q^{\star}\left(\xi_{1}, \xi_{2}\right)$ the condition $\Delta E_{F}<0$ yields $\Delta S_{q}>0$. This is 
so because in this cases $\Delta E_{F}$ and $\Delta S_{q}$ can be written under the form of integrals in the interval $\left[\xi_{1}, \xi_{2}\right]$ with integrands always negative and positive, respectively. In case (b) we adopt the same procedure which yields, in this case, the auxiliary function of Eq. (26):

$$
q^{\star \star}(\xi) \equiv \max \left\{1, \alpha_{1}^{\star \star}(\xi), \alpha_{2}^{\star \star}(\xi), \alpha_{3}^{\star \star}(\xi)\right\} .
$$

As to the term $\alpha_{j}^{\star \star}(\xi)$, in the case where the constraints

$$
P_{j}(\xi)>0 \quad \text { and } \quad d P_{j} / d \xi<0
$$

hold true, we set

$$
\alpha_{j}^{\star \star}(\xi) \equiv 1+\left[\ln \left(\frac{P_{m}}{P_{j}}\right)\right]^{-1} \ln \left[3\left(\frac{d P_{m}}{d \xi}\right)^{-1}\left|\frac{d P_{j}}{d \xi}\right|\right] .
$$

If the constraints of the Eq. (A10) do not apply, we set $\alpha_{j}^{\star \star}(\xi)=1$. The counterpart of Eq. (23) becomes Eq. (24):

$$
Q^{\star \star}\left(\xi_{1}, \xi_{2}\right) \equiv \sup _{\xi \in\left[\xi_{1}, \xi_{2}\right]}\left\{q^{\star \star}(\xi)\right\} .
$$

In this case, for $q>Q^{\star \star}\left(\xi_{1}, \xi_{2}\right)$ the condition $\Delta E_{F}>0$ yields $\Delta S_{q}<0$. This concludes the demonstrations of the properties described in Sec. III D.
[1] C. H. Bennett, G. Brassard, C. Crépeau, R. Josza, A. Peres, and W. K. Wootters, Phys. Rev. Lett. 70, 1895 (1993).

[2] S. Hill and W. K. Wootters, Phys. Rev. Lett. 78, 5022 (1997).

[3] S. Popescu, Phys. Rev. Lett. 72, 797 (1994).

[4] M. B. Plenio and V. Vedral, e-print quant-ph/9804075.

[5] S. Popescu, D. Rohrlich, Phys. Rev. A 56, R3319 (1997).

[6] P. Horodecki, R. Horodecki, M. Horodecki, Acta Phys. Slov. 48, 141 (1998).

[7] N. J. Cerf and C. Adami, Phys. Rev. Lett. 79, 5194 (1997).

[8] C. Brukner and A. Zeilinger, e-print quant-ph/0008091.

[9] C. Brukner and A. Zeilinger, e-print quant-ph/0006087.

[10] C. Brukner and A. Zeilinger, Phys. Rev. Lett. 83, 3354 (1998).

[11] C. Tsallis, J. Stat. Phys. 52, 479 (1988).

[12] S. Abe, Phys. Lett. A 271, 74 (2000).

[13] C. Tsallis, Braz. J. Phys. 29 (1999).

[14] A. Vidiella-Barranco, Phys. Lett. A 260, 335 (1999).

[15] S. Abe and A. K. Rajagopal, Phys. Rev. A 60, 3461 (1999).

[16] S. Abe and A. K. Rajagopal, e-print quant-ph/0001085.

[17] C. Tsallis, S. Lloyd, and M. Baranger, e-print quant-ph/0007112.
[18] E. Jaynes, Phys. Rev. 108, 171 (1957).

[19] E. Jaynes, Phys. Rev. 108, 620 (1957).

[20] W. K. Wootters, Phys. Rev. Lett. 80, 2245 (1998).

[21] C. H. Bennet, D. P. DiVincenzo, J. Smolin, and W. K. Wootters, Phys. Rev. A 54, 3824 (1996).

[22] R. Horodecki and M. Horodecki, Phys. Rev. A 54, 1838 (1996).

[23] C. H. Bennett, G. Brassard, S. Popescu, B. Schumacher, J. A. Smolin, and W. K. Wootters, Phys. Rev. Lett. 76, 722 (1996).

[24] R. F. Werner, Phys. Rev. A 40, 4277 (1989).

[25] D. Giulini, E. Joos, C. Kiefer, J. Kupsch, I.-O. Stamatescu, and H. -D. Zeh, Decoherence and the Appearance of a Classical World in Quantum Theory (Springer-Verlag, Berlin, 1996).

[26] L. Tessieri, D. Vitali, and P. Grigolini, Phys. Rev. A 51, 4404 (1995).

[27] R. Horodecki, M. Horodecki, and P. Horodecki, Phys. Rev. A 59, 1799 (1999).

[28] A. Wehrl, Rev. Mod. Phys. 50, 221 (1978).

[29] A. K. Rajagopal, e-print quant-ph/9903083.

[30] M. Horodecki, R. Horodecki, Phys. Lett. A 244, 473 (1998). 